\title{
Saúde mental: concepções e práticas de agentes comunitários de saúde da cidade de Joinville
}

\section{Mental health: conceptions and practices of community health agents of Joinville city}

\author{
Ana Carolina Schmidt* \\ Bianca Berns* \\ Gislaine Sergio* \\ Marina LeHMANN* \\ VAnessa Cristina Ehrlich* \\ Sofia Cieslak Zimath ${ }^{* *}$
}

\section{Resumo}

Este estudo investigou as concepções e práticas em saúde mental de agentes comunitários de saúde da cidade de Joinville. A pesquisa quali-quantitativa foi realizada com 40 ACS de cinco unidades básicas de saúde da cidade. Os resultados apontaram que $80 \%$ dos participantes não se sentem preparados para a demanda relativa à saúde mental. $\mathrm{O}$ entendimento de saúde mental dos agentes comunitários foi orientado pelo conceito da doença em $67 \%$ das respostas. Foi possível observar práticas endereçadas ao encaminhamento médico. Por outro lado, a preocupação com uma prática humanizada também foi expressada. A concepção de saúde mental marcada pela questão

* Acadêmicas do curso de graduação em Psicologia da Universidade da Região de Joinville - UNIVILLE; Trabalho realizado em Estágio Supervisionado.

** Orientadora, professora do curso de Psicologia da Universidade da Região de Joinville - UNIVILLE. 
da doença deve ser compreendida em sua relação com a sociedade. Além disso, indica a necessidade de capacitação na área.

Palavras-chave: Atenção Básica, Agentes Comunitários de Saúde, Saúde Mental

\begin{abstract}
The article sought to identify the conceptions and practices in mental health of the community health agents of the city of Joinville. The qualitative-quantitative research was performed with $40 \mathrm{CHAs}$ from five basic health units of the city.. The results indicated that $80 \%$ of the participants did not feel prepared for the demand related to mental health. The mental health understanding of community agents was guided by the concept of the disease in $67 \%$ of the responses. It was possible to observe practices addressed to medical referral. On the other hand, the concern with a humanized practice was also expressed. The conception of mental health marked by the issue of illness must be understood in its relation with society. In addition, it indicates the need for capacity building in the area.
\end{abstract}

Key words: Primary Care, Community Health Agents, Mental Health

\title{
Introdução
}

Ao longo da história, a saúde mental foi concebida de diferentes formas, influenciando as práticas dos profissionais da área. Existem muitas definições de saúde, levando em consideração questões estatísticas, funcionais, subjetivas (DALGALARRONDO, 2011).

No Brasil, o atendimento público de saúde é realizado pelo Sistema Único de Saúde - SUS, uma conquista advinda de reivindicação pública, criado pela Constituição de 1988 (BRASIL, 2009). Dois conceitos fundamentais do SUS são o de território e rede. As políticas nacionais buscam incluir o indivíduo como sujeito social, que vive em uma rede social. Partindo do território de moradia do sujeito, os profissionais da saúde iniciam seu olhar sobre o mesmo pois é onde os sintomas emergem (ZURBA, 2011, p. 8). Dessa forma, o sujeito imerso em um território dispõe de uma rede de saúde, composta pela atenção básica, de média complexidade e de alta complexidade, que incluem unidades básicas de saúde, Centros de Atenção Psicossocial (CAPs) e hospitais, respectivamente. 
Nesse sentido, o agente comunitário de saúde - ACS apresenta-se como profissional essencial no primeiro contato com a comunidade, no nível de atenção básica, destacando-se uma de suas ações específicas: "desenvolver atividades de promoção da saúde, de prevenção das doenças e agravos e de vigilância à saúde, por meio de visitas domiciliares e de ações educativas individuais e coletivas nos domicílios e na comunidade" (BRASIL, 2012, p. 49).

Percebe-se que é crucial que as concepções de saúde mental desses profissionais sejam qualificadas. Nesse contexto, a pesquisa pretendeu identificar as concepções e práticas em saúde mental, por parte dos agentes comunitários de saúde da cidade de Joinville.

\section{Concepções e práticas em saúde mental ao longo da história}

Consideramos importante um breve resgate histórico das concepções e práticas em saúde mental. Nesse sentido, Silveira e Braga (2005) entenderam que analisar como a saúde mental foi tratada permite "desnaturalizar" conceitos e refletir sobre as ações em saúde mental.

Podemos estabelecer o século XVII como marco, visto que o internamento de pessoas em sofrimento psíquico se estabeleceu como forma de afastar da sociedade quem ferisse ou incomodasse os padrões de condutas sociais (SANT'ANNA; BRITO, 2006)

No século XVII o louco era marginalizado socialmente, após esse cenário aparecem os hospitais que passou a realizar uma função social e política. Muitos médicos migraram para esse espaço, com o objetivo de "curar enfermos" (AMARANTE, 2007). Torres e Amarante (2011) comentam que Foucault entendera que o médico tomou posição de "mestre da loucura", existindo uma produção da doença mental pelo poder médico. Esse poder de intervenção médica começou a ser desmistificado, segundo Batista (2014) somente a partir da necessidade de diminuir a superlotação dos manicômios e gastos públicos. E, de acordo com Hirdes (2009) apenas no século XX surgiu o movimento visando a des institucionalização como crítica epistemológica ao saber médico da psiquiatria. Conforme Amarante (1995, apud SILVEIRA; BRAGA, 2005) a reforma italiana, liderada por Franco Basaglia, questionou o conceito de doença mental, no 
sentido de identificar a "existência-sofrimento" do sujeito em relação com o corpo social.

No final dos anos 1970, chegaram ao Brasil as ideias de Basaglia - que junto à ideias de Foucault, segundo Torres e Amarante (2011) - inspiraram a reforma psiquiátrica brasileira. É um marco do movimento dos trabalhadores em saúde mental, a promulgação da Lei Federal no 10.216/01, de abril de 2001, conhecida como Lei Antimanicomial, da qual se destaca o respeito à cidadania e atenção integral no processo de saúde/doença mental, relacionando-o diretamente com qualidade de vida (SILVEIRA; BRAGA, 2005). Ressalta-se, ainda, que as ações em saúde mental precisam estar onde os indivíduos estão" (HIRDES, 2009). Dessa forma, a inserção de tais ações no SUS, possuem importância salutar.

Ante tais considerações, o presente estudo teve como objetivo investigar qual a compreensão de agentes comunitários acerca do conceito e de suas funções em saúde mental.

\section{Método}

A pesquisa caracteriza-se como exploratória com abordagem quali-quantitativa. A perspectiva exploratória se deu em função da falta de estudos sobre o tema, que para Creswell (2010) este tipo de pesquisa possibilita entender os significados atribuídos a um problema social pelas pessoas. A abordagem quantitativa, de acordo com Silveira e Gerhardt (2009), é concentrada na objetividade, inicia o processo com ideias preconcebidas pelo qual os conceitos estão relacionados e analisa os dados numéricos através de procedimentos estatísticos. Já a pesquisa qualitativa, conforme Marconi e Lakatos (2017) servirá como base para a compreensão do fenômeno dentro de um contexto.

Participantes: participaram 40 agentes comunitários de saúde. Entre esses agentes contaram-se 1homem e 39 mulheres, em faixa etária que variou de 30 a 60 anos de idade, com formação variando do ensino fundamental até ensino superior completo. Os agentes atuavam em cinco unidades básicas de saúde do município, sendo três localizadas na região norte e duas na região sul. Foram incluídos os agentes concursados que se dispuseram a participar e que estavam presentes na data em que os dados foram coletados. A 
exclusão se deu pelo impedimento de participação em função das atividades no dia da coleta de dados.

Material e Instrumento: o instrumental para recolhimento de dados foi um questionário. O mesmo, elaborado pelo grupo de pesquisa, foi composto por duas sessões, uma delas para o levantamento do perfil dos participantes (identificou a idade, o sexo, tempo de atuação na área da saúde e formação acadêmica). A segunda sessão do instrumento continha questões abertas e fechadas para mapeamento das funções do agente comunitário de saúde, a concepção e as ações relacionadas à saúde mental e preparo para atuação neste campo.

Ambiente: a investigação foi realizada nos próprios locais de trabalho dos participantes, ou seja, nas Unidades de Saúde, em sala cedida pela instituição (sala de reuniões da unidade).

Procedimento: a pesquisa foi devidamente aprovada pelo Comitê de Ética em Pesquisa da Universidade, sob parecer $\mathrm{n}^{\circ} 2.180 .672$ de 20/07/2017. A pesquisa também levou em consideração as orientações do Conselho Federal de Psicologia, dispostas na Resolução CFP16/00, que dispõe sobre a realização de pesquisa em Psicologia com seres humanos.

A viabilidade da pesquisa se deu em função da aceitação da Secretaria da Saúde que forneceu a carta de anuência concordando com a realização do estudo. As pesquisadoras entraram em contato com os ACS (Agentes Comunitários de Saúde) após terem realizado o contato com os responsáveis das cinco UBS (Unidade Básica de Saúde) escolhidas, ocasião em que se verificou o melhor período para a coleta de dados.

Os ACS, que se prontificaram a participar da pesquisa foram abordadas em grupo, em uma sala de reuniões da unidade, tomando conhecimento do Termo de Consentimento Livre e Esclarecido (TCLE) e expressando a concordância com o mesmo através da assinatura deste termo. Em seguida foram distribuídos os questionários a cada ACS e as pesquisadoras permaneceram na sala para esclarecerem as dúvidas dos participantes. Para garantir o sigilo sobre a identificação dos participantes não houve necessidade de nomear o questionário, esses foram apenas numerados. Após o preenchimento do questionário este era depositado num envelope; posteriormente as questões 
fechadas foram digitadas em Excel e analisadas por estatística simples; respostas às questões abertas foram analisadas por categoria de análise a partir do conteúdo e da literatura acerca de saúde/doença mental.

\section{Resultados e discussão}

Com base na análise do conteúdo extraído, foram criadas 4 categorias de análise: "Atribuições de um Agente Comunitário de Saúde"; "Concepção de Saúde Mental"; "Experiências Profissionais em Saúde Mental"; "Conhecimentos e habilidades". Passamos a apresentá-las e discuti-las a seguir.

\section{As atribuições de um Agente Comunitário de Saúde}

Os participantes da pesquisa foram questionados sobre quais seriam as principais atribuições de um Agente Comunitário de Saúde (referindo-se à saúde geral), por ordem de importância. A partir da coleta de dados, as cinco atribuições mais indicadas pelos participantes foram as seguintes: orientação, acompanhamento, cadastro, visita e prevenção - conforme exposto no Gráfico 1:

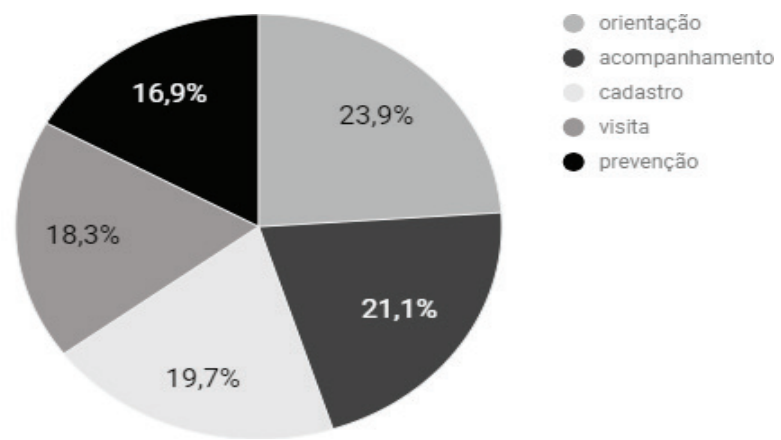

Figura 1: Atribuições dos Agentes Comunitários de Saúde

Foi possível perceber (conforme Figura 1) que os ACS estavam familiarizados com suas atribuições (Orientação, acompanhamento, cadastro, visitas, prevenção), indo ao encontro com o que é descrito pela Política Nacional de Atenção Básica do Ministério da Saúde (BRASIL, 2012): ao ACS compete realizar o cadastro das famílias, orientá-las quanto à utilização dos serviços de saúde, acompanhá-las através de visitas domiciliares e desenvolver atividades de promo- 
ção e prevenção em saúde. Além das respostas mais recorrentes apresentadas, foram citadas como atribuições a questão do vínculo, ou seja, a conexão entre unidade de saúde e comunidade, além de promoção de atividades em grupo.

Por outro lado, quando questionados sobre as atribuições de um ACS na área específica de saúde mental, alguns participantes da pesquisa expressaram confusão em suas respostas, não apresentando a mesma familiaridade que tinham com a "saúde geral, citando a realização de diagnóstico como uma atribuição, por exemplo. Diante disso, depreende-se que a saúde mental é dissociada quanto a integralidade da saúde, por parte destes profissionais. Não parece existir o entendimento que há um sofrimento subjetivo associado a toda doença. Porém, conforme Delgado et al (2007, p. 64) propõem, “poderíamos dizer que todo problema de saúde é também - e sempre - de saúde mental, e que toda saúde mental é também - e sempre - produção de saúde".

Portanto, o entendimento da saúde de forma integral é uma questão a ser tratada com os profissionais da área, visto que se faz fundamental a articulação entre saúde mental e atenção básica, principalmente considerando o avanço nesses campos (OLIVEIRA, 2015).

\section{Concepção de Saúde Mental}

Os Agentes Comunitários de Saúde responderam o que entendiam por saúde mental. As respostas foram agrupadas em 3 categorias, conforme é possível observar na figura 2. A concepção de saúde mental de $67 \%$ dos participantes mostrou-se enviesada pelo conceito de doença. Por outro lado, 18\% dos Agentes Comunitários de Saúde relacionaram saúde mental a uma questão de equilíbrio e/ ou controle. O restante das respostas apresentou conteúdos diversos.

Com isso é possível dizer que as concepções e práticas para com a saúde mental apresentaram grande avanço proporcionado pela reforma psiquiátrica; as estratégias do SUS (Sistema Único de Saúde do Brasil), como a de Saúde da Família - ESF - despontaram com a incumbência de fortalecer essa nova perspectiva de saúde mental. Porém, muitos profissionais ainda possuem como referência a concepção de doença mental, conforme também já mencionou Oliveira (2015). Neste sentido, também Andreoli (2007) destaca que um dos grandes desafios de saúde mental é justamente qualificar os profissionais a ter visões mais humanas dos pacientes. 


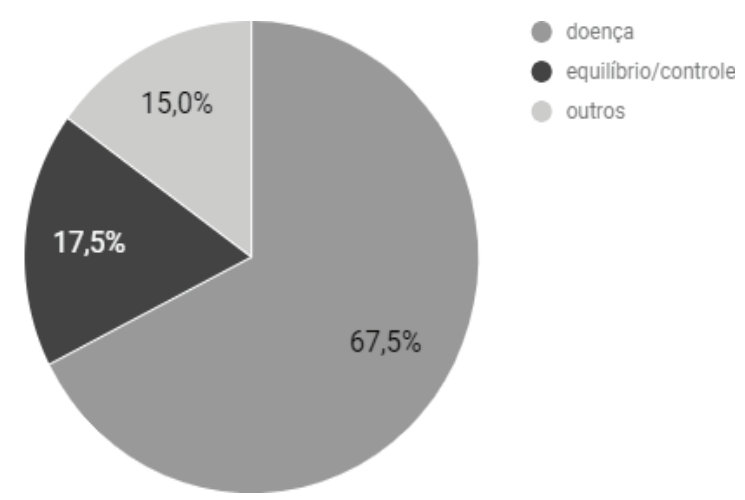

Figura 2: Concepção de Saúde Mental

Também foi possível observar que as respostas envolvendo a concepção de doença, assim como aquelas relacionadas ao equilíbrio/ controle, partiram de princípios diferentes: transtorno, tratamento medicamentoso, incapacidade e dificuldade de resolver problemas, normalidade, entre outros.

Dessa forma, vale destacar algumas respostas sobre o entendimento de saúde mental como sendo "paciente ou a pessoa que sofre de algum modo por algum problema como depressão, fobia, transtorno e outros que usam na sua maioria medicação controlada para poder manter uma forma digna de vida com menos sofrimento" (Participante $\mathrm{n}^{\circ}$. 24). Outro participante segue o mesmo pensamento, "Saúde mental entendo que é o tratamento feito com as pessoas que tem algum tipo de desequilíbrio tipo: depressão, ansiedade, transtorno, etc" (Participante n‥9).

É possível observar que o pensamento que compreende saúde mental pelo viés da doença é o mesmo que concebe os serviços de saúde pela perspectiva do tratamento. É importante lançar uma reflexão sobre essa percepção com as contribuições de Giovanella e Amarante (1994) que pôde apontar para o fato de que, ao partir do conceito de doença mental, a psiquiatria estabeleceu as concepções de cura e tratamento, mas ambas podem ser desconstruídas. Assim, o foco da doença e o foco do processo de cura, podem dar lugar ao processo de invenção da saúde e reprodução social dos sujeitos. Entretanto, há de se observar, que os dizeres de Amarante foram expressos em sua obra no ano de 1994, ou seja, há 23 anos; o que parece que ainda continuam arraigadas as antigas concepções, nos próprios profissionais de saúde. 
Um dos participantes se posicionou de maneira diferente das respostas apresentadas anteriormente: "Saúde mental não é apenas não estar doente, é talvez encontrar uma maneira de vida saudável dentro das necessidades que encontram, que é saber lidar com a situação" (P30). Nesta resposta a saúde não foi associada à ideia de doença/ou tratamento e, ainda, o conceito de doença parece não estar separado do contexto social do indivíduo. É importante que este tipo de concepção faça parte do repertório dos profissionais em saúde, de forma que as abordagens para com a saúde mental não existam apenas associadas a conhecimentos e espaços médicos e psicológicos, mas a todos os espaços de integração e vivência dos sujeitos, conforme apregoou Dell'Acqua (1987, apud GIOVANELLA; AMARANTE, 1994).

Vale salientar que o aparato manicomial não se trata apenas de hospitais psiquiátricos, mas, trata-se também de relações de preconceito, de poder e dominação. Há uma hierarquia que, segundo Giovanella e Amarante (1994) se baseia na possibilidade ou não de se adaptar ao que socialmente é dito normalidade. Portanto, ao não problematizar tais questões, contribui-se para a solidificação dos conceitos de anormalidade mental, concepções de adaptação e ideias de saúde mental, de tal forma que esses três tópicos estiveram presentes nas respostas de grande parte dos participantes. Acerca da anormalidade, um dos ACS respondeu: "É tudo que compete ao indivíduo ao seu ser capaz de compreender as ações tomadas se são ou não normal" (Participante no. 22). Além da questão da normalidade, se infere também a responsabilização do sujeito frente a sua saúde.

Sobre a adaptação/desadaptação enquanto critério para a saúde mental houve respostas relacionadas a esse conceito tanto na categoria de doença, como na categoria de equilíbrio/controle. Um dos participantes declarou como entendimento de saúde mental, o seguinte: "Pessoas que têm alguma dificuldade em se compor no meio de outras pessoas, em caso de algum problema; quem tem algum problema necessita de ajuda!" (Participante $\mathrm{n}^{\mathrm{o}}$. 36). Destaca-se aqui, conforme já apontara Jahoda (1958 apud OLIVEIRA, 2015) que na medida em que a OMS elaborava um conceito de saúde que envolvia a concepção de completo bem-estar físico, mental e social, em 1947 Karl Menninger definia saúde mental como uma forma de adaptação do sujeito ao mundo e aos outros com efetividade e uma disposição alegre, tendo consideração social. 
Dessa maneira, há décadas a saúde mental vem sendo associada à adaptação social, de forma que, conforme Oliveira (2015), o questionamento existencial sobre a saúde mental é desautorizado pela sociedade. Porém, continua o mesmo autor, vivendo-se em um sistema socioeconômico capitalista que se fundamenta na desigualdade, com altos índices de desemprego, violência, entre outros aspectos, como não apresentar angústia, independentemente da posição social ocupada? Como adaptar-se alegremente a esses fatores?

A discussão desse tipo de representação se faz necessária, uma vez que as práticas em saúde mental não podem se fundamentar em padrões morais. Este texto não pretende entrar nesta questão propriamente dita, mas cabe levantar questionamentos para pesquisas futuras, ou seja, na presente investigação, foram recorrentes as respostas que relacionaram saúde mental à questão da dependência em drogas e também à estruturação familiar. Mas de que droga os participantes estavam discorrendo? Quais medicamentos estão inclusos nessa categoria? E que tipo de família é idealizada pelo profissional? Assim, reitera-se que drogas, sexualidade, religiosidade, situações financeiras e demais temas, devem ser trabalhados sob a ótica da saúde e não por juízo de valor (ainda bastante impregnado nas concepções sobre saúde/doença entre os próprios profissionais).

Por fim, vale salientar que a reforma psiquiátrica brasileira tem sido sinônimo de luta por direitos relativos à saúde mental e, conforme Oliveira (2015), é importante não se perder de vista a discussão constante do que representa aquilo que chamamos de doença mental e o que podemos chamar de saúde mental.

\section{Experiências Profissionais em Saúde Mental}

Os participantes da pesquisa foram indagados sobre as suas experiências junto a indivíduos com doença mental e emitiram as seguintes respostas: $90 \%$ afirmou ter realizado contato profissional com uma pessoa com doença mental, ao passo que $10 \%$ relatou não ter tido experiência em tal situação. Andreoli (2007) destaca que entre os indivíduos que buscam atendimento médico, na rede de atenção primária no Brasil, a prevalência de transtornos mentais menores está entre 41 a 55\%, ao passo que a prevalência de transtornos mentais graves atinge entre 25 a $38 \%$, aproximadamente. 
Dentre os participantes que responderam já tiveram experiências profissionais que envolveram uma doença mental, as formas de agir foram diversificadas. Apenas 5\% dos agentes demonstrou, em suas respostas, domínio acerca da rede de saúde. É válido destacar um dos relatos em que o participante descreveu o caso de um jovem atendido: "Não saía de casa, não se alimentava, abandonou o emprego, estudos. Não falava com ninguém e não saía de casa, hoje com 20 anos ainda continua, mas se alimenta. Comunicado ao responsável na UBS e encaminhado ao CAPS" (Participante n⿳o. 3). Essa declaração demonstra o conhecimento do ACS acerca da rede de saúde, não apenas pelo entendimento acerca do Centro de Atenção Psicossocial, mas também por contrastar com outras respostas que apresentam o médico como figura principal nesta rede, tal como a seguinte "Segui instruções médicas" (Participante no. 4). Ressalta-se que o participante 3 utilizou o termo "responsável", que pode ser o coordenador da unidade, ou outro profissional. Com isso, entende-se, que tal ação é necessária para que práticas que alienam o indivíduo ao conhecimento médico/ psicológico não sejam reproduzidas.

Muitos participantes foram evasivos em relação às suas práticas em saúde mental, contabilizando $15 \%$ de respostas, descrevendo a ação junto ao indivíduo com doença mental como "normal e/ou naturalmente", o que pode indicar uma insegurança quanto às suas práticas nas situações vivenciadas.

Boa parte das experiências narradas também relacionou doença mental a ideia de surto. Além disso, o sentimento do medo ficou evidente nos relatos. Um dos participantes contou: "Reagi com muita cautela, mas fiquei apreensiva já que a pessoa me abraçou em uma das visitas domiciliares e não largava mais, precisava o familiar interferir, ela me pegou de surpresa" (Participante no. 9). Outro participante afirma: "No início assustada, após tentei conversar com a pessoa até ela se acalmar, não havendo melhora foi chamado assistência médica" (Participante no . 36). Giovanella e Amarante (1994) explicam que no processo de isolamento da loucura, a psiquiatria modifica a experiência da sociedade para com a mesma, e esta aprende a pensar doença mental de forma negativa, relacionada a desumanidade e a periculosidade.

Assim, observaram-se dados desta pesquisa que apontaram para práticas em saúde mental sob a ótica da doença mental e rela- 
cionando o ato em saúde com o conhecimento médico e tratamento medicamentoso, de forma que o princípio de regionalização - que significa, de acordo com a Escola Nacional de Saúde Pública Sérgio Arrouca $(2014$, web) "um processo técnico-político relacionado à definição de recortes espaciais para fins de planejamento, organização e gestão de redes de ações e serviços de saúde" - conforme mencionaram Giovanella e Amarante (1994), ao invés de aproximar, tornou-se método de detecção da doença mental. Por outro lado, alguns ACS narraram práticas relacionadas à escuta qualificada. De acordo com Maynart et al (2014) a escuta qualificada envolve relações de diálogo, vínculo e acolhimento. Também oportuniza a compreensão do sofrimento psíquico a partir da pessoa. De modo geral, configura-se como um desafio fomentar práticas que não venham a reduzir a compreensão de saúde mental com a doença e tampouco limitar o tratamento ao recurso do medicamento, além de restringir o saber em saúde mental ao profissional médico.

\section{Conhecimentos e habilidades}

Em relação às experiências pessoais, constatou-se que $72 \%$ de ACS participantes tiveram contato pessoal com pessoas com transtornos mentais. De acordo com informações de da Organização Mundial da Saúde - OMS (WORLD HEALTH ORGANIZATION, 2011) os transtornos mentais totalizam $20,3 \%$ do total de doenças. $\mathrm{Na}$ presente investigação, apesar das vivências pessoais relatadas, 72,5\% dos participantes declarou possuir pouco conhecimento sobre saúde mental, assim como mostraram necessidade de receber capacitações envolvendo a temática. Quando questionados se na região em que atuam existe uma demanda forte de pessoas com problemas de saúde mental, $78 \%$ responderam positivamente e $22 \%$ responderam de forma negativa. As formas como detectaram essa informação dentro da comunidade foram: pela observação, pelas visitas que realizam frequentemente e no contato com a família. Conforme dito por Andreoli (2007), a importância de que os profissionais da atenção básica possam também identificar demandas de sofrimento psíquico pode auxiliar, por exemplo, na possibilidade de criação de novos Centros de Atenção Psicossocial (CAPS), que vai ser indicada de acordo com o mapeamento dessa necessidade na população... 
Seguindo os resultados, observou-se ainda que, as respostas acerca de "quais conhecimentos e habilidades são importantes para atuação do ACS", originaram 8 sub-categorias conforme tabela 1.

Tabela 1 - Conhecimentos e habilidades de Agentes Comunitários de Saúde

\begin{tabular}{l|c}
\hline \multicolumn{1}{c|}{ SUB- CATEGORIAS ANÁLISE } & PERCENTUAL \\
\hline 1) Necessidade de treinamentos sobre saúde mental & 72,5 \\
\hline 2) Carência de capacitações práticas & 37,5 \\
\hline 3) Afeto pela profissão e a paciência & 12,5 \\
\hline 4) Saber ouvir e ter empatia & 10,0 \\
\hline 5) A própria saúde mental & 7,5 \\
\hline 6) Fazer a ponte entre UBS e comunidade & 5,0 \\
\hline 7) Necessidade de uma remuneração compatível & 5,0 \\
\hline 8) Ausência de habilidades ou conhecimentos necessários para ser um ACS & 5,0 \\
\hline
\end{tabular}

Novamente se identifica a necessidade de treinamento (itens 1 e 2 da tabela 1), tanto prático quanto teórico sobre a temática da saúde mental. Também se mostram relevantes respostas que apontam para um cuidado com uma prática mais humanizada (itens 3 e 4). Neste sentido, é possível recorrer a Giovanella e Amarante (1994) ao explicar que essas práticas estão principalmente relacionadas com a forma de lidar com pessoas, além dos aspectos relacionais em si mesmos. $\mathrm{O}$ item 5 mostra um aspecto importante a ser frisado, o profissional que atua com saúde mental não pode deixar de cuidar da sua própria saúde mental, além de que a clareza da importância de se cuidar pode ser um bom começo.

Algumas das respostas coletadas indicaram, também, a ausência de habilidades ou conhecimentos necessários para ser um ACS (item 8 , tabela 1), tais dados elucidam principalmente o sentimento de despreparo teórico-prático para lidar com demandas relacionadas à saúde mental. Ademais, se mostra intrigante o entendimento de que não há quaisquer habilidades e conhecimentos necessários para exercer a função de ACS.

Sobre o que o ACS deve exercer no campo da saúde mental, distinguiram-se 10 sub-categorias nas respostas, apresentadas na tabela 2. 
Tabela 2 - O ACS na saúde mental

\begin{tabular}{l|c}
\hline \multicolumn{1}{c|}{ SUB-CATEGORIAS DE ANÁLISE } & PERCENTUAL \\
\hline 1) colher informações e levar ao posto & 27,5 \\
\hline 2) orientação para paciente ou familiares & 17,5 \\
\hline 3) acompanhamento do paciente e das medicações & 17,5 \\
\hline 4) encaminhamento do paciente aos locais especializados & 17,5 \\
\hline 5) promoção de trabalhos alternativos, com artes & 12,5 \\
\hline 6) acolhimento e escuta do usuário & 10,0 \\
\hline 7) indicação de trabalho multiprofissional utilizando-se das especialidades da UBS & 7,5 \\
\hline 8) grupos de conversa e apoio com pessoas com transtornos e familiares & 5,0 \\
\hline 9) realizar o diagnóstico do paciente & 2,5 \\
\hline 10) o sentimento de impotência para atender a demanda de saúde mental & 15,0 \\
\hline
\end{tabular}

A partir desses dados, é possível ressaltar alguns aspectos, como a necessidade de treinamentos que satisfaçam à demanda relacionada a saúde mental, conforme enuncia o item 10 da tabela 2. Essa necessidade dos participantes torna-se visível, pois $20 \%$ se sente preparado para atender a demanda e $80 \%$ não; ao mesmo tempo em que as capacitações que realizaram foram escassas para a real necessidade do tema. Dos 40 Agentes investigados, 19 não tinham realizado nenhum treinamento e 21 já tiveram; dentre os treinamentos, a maioria foi realizada pelo CAPS e as outras 14 respostas foram diversas. Importante perceber que grande parte das respostas foi negativa em relação aos treinamentos. Nesse sentido, observam-se também compatibilidade com o dito por Ballester et al. (2005, apud ANDREOLI, 2007) em que há pouca atenção ao treinamento dos profissionais de saúde mental que não são especializados, inclusive o clínico geral que tem seu diploma de medicina, por diversas vezes, precisa fazer encaminhamentos ao especialista.

Ao mesmo tempo em que grande parte dos ACS demonstrou essa preocupação com treinamentos, houve uma porcentagem baixa, mas não excludente, de percepção da função de ACS atrelada ao diagnóstico de patologias (item 9, da tabela 2). Esse é um ponto importante a ser desmistificado por profissionais, uma vez que não haverá preparo para lidar com essa demanda, a qual não compete ao agente comunitário de saúde. Questiona-se o quão claro estão para os ACS quais são suas reais atribuições, dispostas na Portaria no ${ }^{\mathbf{0}}$ 1886/ GM, de 18 de dezembro de 1997 (BRASIL, 1997). Também se torna 
importante observar que a atuação do ACS deve aproximar a rede de saúde e a comunidade, e não ampliar a detecção de desadaptados (GIOVANELLA; AMARANTE, 1994), rotulando esses indivíduos.

Contudo, percebe-se também que o pensamento de práticas humanizadas (itens 5, 6 e 8, da tabela 2) faz-se presente mais uma vez, e essa é uma questão importante, pois os ACS realizam as relações comunidade-unidade.

\section{Conclusão}

A presente pesquisa buscou identificar as concepções e práticas em saúde mental de agentes comunitários de saúde da cidade de Joinville, partindo do entendimento que as concepções são construídas socialmente e influenciam as práticas. E os dados apontaram para uma concepção de saúde mental associada à doença, por parte dos ACS. Observaram-se ainda dificuldades na compreensão das funções de um ACS no campo da saúde mental. Verificou-se que esses ACS sentiam necessidade de que houvesse capacitação na área de saúde mental, o que comungou com uma preocupação bastante presente no discurso desses profissionais com uma prática em saúde humanizada.

Reitera-se a importância de que todos os profissionais que compõem a rede de saúde no que concerne à promoção de práticas humanizadas, se distanciem da ideia de saúde mental atrelada à proposta manicomial. Para tanto, futuros estudos podem investigar a concepção de saúde mental existente nos demais profissionais atuantes nas unidades básicas de saúde. Promover o desenvolvimento dos conceitos de saúde mental é também propiciar a evolução de práticas na área; o que se configura como compromisso ético para os profissionais da saúde, independente da formação. Em tempos de ataques às políticas nacionais de saúde mental, não podemos declinar de tais reflexões.

\section{Referências}

AMARANTE, P. Saúde mental e atenção psicossocial. Rio de Janeiro: Editora Fiocruz, 2007.

ANDREOLI, S. B. Serviços de Saúde Mental no Brasil. In: MELLO, M. F.; MELLO, A.A.F; KOHN, R. (Orgs.). Epidemiologia da saúde mental no Brasil. 1. ed. Porto Alegre: Artmed, 2007. 207 p. 
BATISTA, M. D. G. Breve história da loucura, movimentos de contestação e reforma psiquiátrica na Itália, na França e no Brasil. Revista de Ciências Sociais, n. 40, 2014, p. 391-404. Disponível em: <https://goo.gl/mgWgX6>. Acesso em: 21 mai. 2017.

BRASIL. Ministério da Saúde. Portaria GM nº1886, de 18 de dezembro de 1997. Aprova as Normas e Diretrizes do Programa de Agentes Comunitários de Saúde e do Programa de Saúde da Família. Disponível em: <http://189.28.128.100/dab/docs/ legislacao/portaria1886_18_12_97.pdf>. Acesso em 13 out. 2017.

Ministério da Saúde. Secretaria de Atenção à Saúde. Departamento de Atenção Básica. O trabalho do agente comunitário de saúde. Brasília, 2009. Disponível em: <https://goo.gl/EcBk9X>. Acesso em: 09 nov. 2017.

- Ministério da Saúde. Secretaria de Atenção à Saúde. Departamento de Atenção Básica. Política Nacional de Atenção Básica. Brasília, 2012. Disponível em: <http://189.28.128.100/dab/docs/publicacoes/geral/pnab.pdf>. Acesso em: 09 nov. 2017.

BRAZIL. Mental health atlas. Department of mental health and substance abuse, World Health Organization, 2011. Disponível em: <https://goo.gl/63x5SL>. Acesso em 13 out. 2017.

CRESWELL, J. W. Projeto de pesquisa: métodos qualitativos, quantitativo e misto. 3.ed. Porto Alegre: Artmed, 2010.

DALGALARRONDO, P. Psicopatologia e Semiologia dos Transtornos Mentais, 2a edição. ArtMed, 2011.

DELGADO, P.G.G. et al. Reforma psiquiátrica e política de saúde mental no Brasil In: MELLO, M. F.; MELLO, A.A.F; KOHN, R. (Orgs.). Epidemiologia da saúde mental no Brasil. 1. ed. Porto Alegre: Artmed, 2007. 207 p.

ESCOLA NACIONAL DE SAÚDE PÚBLICA SÉRGIO ARROUCA. A regionalização pode contribuir para o avanço do SUS? Disponível em: <http://www.ensp.fiocruz.br/ portal-ensp/informe/site/materia/detalhe/35859>. Acesso em: 24 nov. 2017.

GERHARDT, T.E; SILVEIRA, D.T. Métodos de Pesquisa. Porto Alegre: Editora da UFRGS, 2009. Disponível em: <https://goo.gl/i38C84>. Acesso em: 21 mai. 2017.

GIOVANELLA, L.; AMARANTE, P. O enfoque estratégico do planejamento em saúde e saúde mental. In: AMARANTE, P. (Org.). Psiquiatria social e reforma psiquiátrica. 1a. ed. Rio de Janeiro: FIOCRUZ, 1994. 202 p.

HIRDES, A. A reforma psiquiátrica no Brasil: uma (re)visão. Ciência e saúde coletiva, Rio de Janeiro, v. 14, n. 1, p. 297-305, 2009. 
MARCONI, M. A.; LAKATOS, E.M. Metodologia científica. 7.ed. São Paulo, 2017.

MAYNERT, W. H.C. et al. A escuta qualificada e o acolhimento na atenção psicossocial. Acta Paul Enferm. v. 27, n.4, p.300-3. 2014. Disponível em: <http://www. scielo.br/pdf/ape/v27n4/1982-0194-ape-027-004-0300.pdf>. Acesso em: 24 nov. 2017.

OLIVEIRA, W.F.. O conceito de saúde mental e a evolução do campo da saúde coletiva e da atenção psicossocial. In: ROBERTI JR, J.P; BENETTI, I.C; OLIVEIRA, W.F. (Orgs.). Saúde mental: diálogos ocupacionais e reflexões psicossociais. 1a. ed. Rio do Sul: UNIDAVI/, 2015. cap. 1, p. 15-35.

SANT'ANNA, T. C.; BRITO, V.C.A.. A lei antimanicomial e o trabalho de psicólogos em instituições de saúde mental. Psicologia: ciência e profissão, Brasília, v. 26, n. 3, p. 368-383, 2006. Disponível em: https://goo.gl/igwE9v . Acesso em: 21 mai. 2017.

SILVEIRA, L.C.; B.; BRAGA, V. A. Acerca do conceito de loucura e seus reflexos na assistência de saúde mental. Revista Latino-Americana de Enfermagem, Ribeirão Preto, v. 13, n. 4, p. 591-595, 2005. Disponível em: http://www.scielo.br/scielo. php?script=sci_arttext\&pid=S0104-11692005000400019\&lng=en\&nrm=iso . Acesso em: 21 mai. 2017.

TORRES, E.H. G.; AMARANTE, P. Michel Foucault e a "História da Loucura": 50 anos transformando a história da psiquiatria. Cadernos Brasileiros de Saúde Mental, Florianópolis, v. 3, n. 6, p. 41-64, 2011.

ZURBA, M. C. Contribuições da psicologia social para o psicólogo na saúde coletiva. Psicologia e Sociedade, v. 23, n. especial, p. 5-11, 2011.

Contato dos autores:

E-mails: annnacaroll1@hotmail.com; bernsbianca1@gmail.com; gisguh@gmail.com; marina-lehmann@hotmail.com; vanessa120993@gmail.com.; sofiaczimath@yahoo. com.br

Texto recebido em: 10/12/2017

Aceito em: 05/08/2018 